\title{
Les différentes sortes de pédagogie
}

\author{
Dr. Tarek Saker; Dr : Said Mezroua \\ Université de Alger -3- Biskra, Algérie.
}

\section{Résumé :}

Depuis les années 1980 , le terme «didactique» a été largement utilisé pour identifier les notions relatives à l'acte éducatif dès lors qu'elles auront le trait scientifique. Un débat profondément établi pour définir, déterminer et aussi pour distinguer entre les différents termes qui décrivent l'acte pédagogique «éducationpédagogie-didactique »

منذ الثمانينيات بإمكاننا ان نلاحظ الاستعمال الواسع لمصطلح التعليمية للتعبير عن الأعمال المتعلقة بالفعل التربوي بمجرد ان تأخذ الطابع العلمي. لقد قام جدال عميق من أجل تعريف و تحديد التمييز بين التربية و البيداغوجية والتعليمية في وصف الفعل التربوي. 


\section{Introduction}

L'éducation paraît recouvrir l'acte effectif, l'ensemble des opérations menées par l'enseignant ou le pédagogue en interaction avec les actes des élèves et les structures sociales; elle réclame réflexions et formalisations pour sa propre évolution. En ce sens, elle inclut dans son essence des activités réflexives pédagogiques et didactiques.

Dans ce champ de pratique sociale qu'est l'éducation, $\underline{l a}$ didactique se présente comme l'activité scientifique tentant de se spécifier au sein des sciences et particulièrement des sciences de l'éducation en limitant son objet à une partie des phénomènes présents dans l'acte éducatif. Hebrard (nd) la définir comme « l'étude conjointe des processus d'acquisition chez l'élève et de transmission chez l'enseignant des contenus de l'enseignement ».

La pédagogie, à la fois action et réflexion, serait donc positionnée entre l'acte d'éduquer et les recherches objectives qui s'y développent. Hebrard (nd) la défini comme «une mise en œuvre pratique des résultats des travaux de la didactique (nécessairement théoriques)... il la considérer aussi comme ... une activité réflexive pouvant être qualifiée de scientifique lorsqu'elle étudie les phénomènes non étudiés par la didactique ». Ainsi lorsqu'on étudie tous les phénomènes de présentation des tâches aux élèves, les phénomènes de relation interindividuelle ou lorsqu'on s'interroge sur les attitudes singulières que font naître les pratiques, nous avons 
Revue des sciences de l'homme et de la société.. Dr. Tarek Saker, Dr : Said Mezroua

tendance à classer ces activités sous le terme de pédagogie. Ces études feront plus appel aux méthodes dites cliniques qu'aux méthodes quantitatives chères aux approches expérimentales.

La réflexion didactique semble s'arrêter là où apparaissent les problèmes de l'ordre de l'axiologie ou de la politique. Autrement dit, trois types de problèmes semblent traverser l'éducation : des problèmes didactiques, axiologiques, et politiques. De tels problèmes ne peuvent avoir d'autres conclusions qu'incertaines, incomplètes et provisoires. Cependant, elles nourrissent le quotidien de l'enseignant. C'est au sein de la pédagogie que s'établissent les liaisons nécessaires entre théorie et pratique.

\section{Notion de pédagogie}

C'est dans et par la recherche que le métier de maître cesse d'être un simple métier et dépasse le niveau d'une vocation affective pour acquérir la dignité de toute profession relevant à la fois de l'art et de la science, car les sciences de l'enfant et de sa formation constituent plus que jamais des domaines inépuisables" (Piaget ,1969).

L'exigence de la recherche apparaît très tôt dans la réflexion pédagogique. J.A. Comenius (moitié du XVIIe siècle), J.F. Herbart et A. Cournot (début XIXe) souhaitent déjà une pédagogie expérimentale, mais ce n'est que vers la fin du XIXe siècle, avec les progrès considérables des laboratoires de psychologie expérimentale qu'apparaît avec force l'idée d'une recherche pédagogique considérée 
comme un prolongement de la recherche psychologique. Mais, le pédagogue est peu formé aux techniques de la recherche. Cet état de fait, qui se prolonge paradoxalement jusqu'à nos jours, met le pédagogue en position de dépendance vis à vis du psychologue qui lui, n'a pas la responsabilité de l'action.

Dès que le fait pédagogique est perçu comme multidimensionnel, ne pouvant se réduire au seul fait psychologique, la tutelle s'étend. Elle devient biologique, sociologique et historique. Binet, dès 1911, montre que la recherche pédagogique peut bénéficier des résultats acquis et des méthodes utilisées dans la recherche scientifique. Durkheim (1922) lui fait écho quelques années plus tard : la pédagogie cette "théorie pratique", cette "réflexion rationaliste" qui tient en haleine l'éducation pour l'empêcher de tomber sous le joug des habitudes, doit chercher dans la psychologie et la sociologie, les principes pour la conduite ou la réforme de l'éducation.

La recherche pédagogique à été toujours un phénomène complexe, son champs d'intervention ne se limite pas à l'innovation et à l'amélioration du fait éducatif. La pédagogie comme la souligne le dictionnaire de psychologie «science ayant pour objet l'étude des doctrines et des techniques sur lesquelles sont fondées l'actions des éducateurs, il ajoute aussi que chaque société à un projet pédagogique précis concernant l'éducation intellectuelle morale et physique de ses membres, qui reflète son idiologie plus particulièrement l'homme accompli et celle de l'enfant ». (1996, p. 1458). 
Revue des sciences de l'homme et de la société.. Dr. Tarek Saker, Dr : Said Mezroua

Donc celle-ci variant considérablement dans le temps, dans l'espace et même les méthodes pédagogiques, qui ne sont ni stables ni uniformes, en d'autres termes, les modèles ne sont pas les mêmes en Algérie et en Belgique francophone à l'époque de l'école traditionnelle hérite par le colonialisme et celle de l'école fondamentale.

La pédagogie est une discipline qui évolue aussi avec le progrès de la science étant un carrefour pluridisciplinaire, Pourtant, si simple qu'il puisse paraître, ce concept de pédagogie n'en recèle pas moins une «...ambiguïté caractérisée des fausses notions claires» (Toralle, 1968, p.7). Ce ci fera dire à Gabaude (1972) que la pédagogie est «à la fois science et technique, doctrine et art, technologie de l'enseignement, ou encore, culture » (p.9).

Traditionnelle, dogmatique, nouvelle, institutionnelle, fonctionnelle ou bien non directive sont autant de qualificatifs qui correspondent à des caractéristique ayant été associées à la notion de pédagogie, selon l'angle par lequel elle est appréhendée. L'éventail est large et les difficultés de définition se rattachent à la pluralité conceptuelle. Toutefois, nous allons essayer de clarifier cette notion en cherchant des éléments de réponse dans la littérature qui y est consacrée. 
La pédagogie, en perpétuelle transformation, se modifie et évolue sous le poids de l'histoire des sociétés et en fonction des connaissances des disciplines sur lesquelles elle s'appuie : les sciences humaines (psychologie, psychanalyste, sociologie, etc). Réduite d'abord à un simple transvasement de connaissances d'un adulte vers un enfant, c'est la pédagogie «cruche et pot» que récuse Rogers (1984), communément appelée 'traditionnelle'.

Quand, à la lumière des nouvelles données de la psychologie, elle prend pour point de départ l'enfant, elle devient 'nondirective'. Entre ces deux pôles fortement contrastés qui continuent jusqu'à l'heure actuelle de s'opposer, la pédagogie adopte plusieurs conceptions sous-jacentes selon le courant envisagé.

Aujourd'hui, «la pédagogie, sollicitée par des idéologies diverses et contradictoires, sujette aux avatars d'un monde modulé par les technologies, propose aux maîtres des modèles qui vont du didacticien le plus froid à l'animateur de groupes, en passant par toutes les modulations possibles sur le thème de la liberté créative » (Gilbert, 1980, p.9). Les vocables attribués par les courants idéologiques semblent très diversifiés mais, en dépit des apparentes différences, la plupart des conceptions se rejoignent pour discerner, en fait, deux grands courants pédagogiques : le traditionnel et le nouveau.

\section{1- Pédagogie traditionnelle}

Souvent mise en cause par les défenseurs de la pédagogie nouvelle «Nous n'avons pas caché notre antipathie à l'égard des 
Revue des sciences de l'homme et de la société.. Dr. Tarek Saker, Dr : Said Mezroua

méthodes dogmatiques, à cause du verbalisme où elles se complaisent, de la passivité à laquelle elles condamnent les élèves et de la soumission morale qu'elles leur imposent » soulignent les auteurs du guide pédagogique (p.9).

Entre autre, Paquette (1979), défenseur de la pédagogie ouverte (nouvelle), adresse une critique sévère à l'éducation traditionnelle : " l'élève est un adulte en miniature et il doit subir l'autorité de l'enseignant »(p.21). Le même auteur ajoute que les valeurs premières de ce type d'enseignement sont la mémoire, la volonté d'obéissance à l'autorité. Pour Louanchi (1987),

« c'est un rapport fondamental d'inégalité entre l'élève et son maître à qui il doit se soumettre en toutes circonstances » (p.325).

Donc, le but de la pédagogie traditionnelle est de transmettre les savoirs d'un cerveau plein (enseignant) à un cerveau vide (élève). La pédagogie traditionnelle présente plusieurs caractéristiques à savoir:

\section{- La référence au modèle}

Mettre l'élève dans une situation qui répond à ses préoccupations. Snyders (1975) souligne que « le fondement de l'éducation traditionnelle est l'ambition de conduire l'élève jusqu'au contact avec les très grandes réalisations de l'humanité »(p.15). Eduquer, c'est donc choisir et proposer des modèles qui permettront à 
l'élève de «s'élever ». Craignant que l'expérience directe ne réduise l'activité de l'enfant à de basses difficultés, Château (1968) prône l'œuvre littéraire.

\section{- Le guidage}

Cette position au modèle ne peut se faire, selon les pédagogues ' traditionnels', sans l'intervention du maître. Snyders (1975) affirme que « entre l'enfant et les œuvres... la distance est si grande qu'un médiateur se révèle indispensable » (p. 22).

\section{- Le morcellement de l'activité}

Dans l'apprentissage, la matière est simplifiée, ordonnée, graduée dans la difficulté en un enchaînement conçu par le maître. Gabaude (1972) considère que «le déroulement du processus didactique se fonde, prioritairement, sur une analyse de l'objet à transmettre ... diviser la difficulté, c'est-à-dire l'objet a transmettre, et partir du simple et facile pour aller progressivement jusqu'au plus complexe »(p.14).

- La transmission des connaissances à sens unique avec pouvoir total de l'enseignant

Le maître détient le savoir, l'enfant le reçoit dans un rapport unilatéral. Ainsi que continue à défendre Gabaude (1972), «le rapport pédagogique s'établit sur la base d'une relation interpersonnelle à sens unique; allant du professeur vers l'élève, à l'occasion de la transmission d'un objet (notion ou valeur) » (p. 14). 


\section{- La mémorisation}

Dans ce type de pédagogie, la mémoire de l'élève est sollicitée par l'enseignant, qui lui demande de respecter une assimilation du message reçu.

Donc la pédagogie traditionnelle était méprisable parce qu'elle ne se préoccupant essentiellement d'enseigner aux élèves des recettes permettant de passer avec succès les examens impériaux, ce qui les amenait nécessairement à la conclusion que le profit personnel est la seul fin de l'apprentissage. Comme le souligne Durkheim (revue de l'éducation, 1993, p. 313).

Le contenu de cet enseignement était arbitraire parce que rien n'est était conçu pour répondre aux besoins des élèves en particulier, et à ceux de l'être humain en général. Cet enseignement portait sur les textes hermétiques et classiques, elle était superficielle parce que tout ce qu'on exigeait des élèves était qu'ils mémorisent et imitent les classiques.

La relation entre maître et élève était fondée sur la frayeur, parce que le premier exerçait une autorité absolue sur le second, qui n'était ni autorisé à lui passé des questions, ni libre d'apprendre sur le plaisir.

Les élèves étaient déprimés parce que la pression exercée sur eux pour qu'ils apprennent était si forte que l'école leur apparaît. 


\section{2- Pédagogie nouvelle}

Elle prend naissance à partir des contestations des méthodes traditionnelles, jugées en inadéquation avec les exigences du temps moderne et sur la base d'une meilleure connaissance de l'enfant (apport de la psychologie génétique). Les principes de cette pédagogie se résument par quatre aspects :

\section{- L'intérêt et le besoin de l'élève}

Ils constituent le point de départ de l'enseignement (on l'appelle communément pédagogie de l'intérêt ou pédagogie fonctionnelle). Le besoin de se développer et de se perfectionner suffit à provoquer et maintenir l'activité de l'enfant, comme cela a été suggéré entre autre, par Montessori (1952), Dewey (1967) et Claparede (1973). L'intérêt véritable de l'activité dirige la conduite de l'enfant, non plus l'enseignant et son programme.

A titre d'exemple, reprenons la thèse de Dewey (1967): « l'intérêt pour le gribouillage doit être mis en œuvre tout de suite et il faut en tirer tout le bien possible sans tarder, sans s'occuper surtout de fait que dans 10 ans, l'élève aura à tenir des livres » (p.83).

\section{- L'enfant comme un être spécifique}

Celui-ci n'est plus considéré comme un «adulte en miniature ». La pédagogie contemporaine, à la lumière des données scientifiques, lui reconnaît son statut propre, même s'il est en évolution. Dans cette optique, Snyder (1975), maintient que 
Revue des sciences de l'homme et de la société.. Dr. Tarek Saker, Dr : Said Mezroua

«l'enfance possède une signification et une visée propres; en ellemême, elle a sa valeur. L'enfant n'est pas un être imparfait, incapable, insuffisant; il réalise une configuration originale et cohérente »(p.56).

\section{- La liberté, l'initiative, l'activité et le travail en groupe}

On retrouve sous ces principes les pédagogies connues sous les vocables 'non directive', 'Freinet', 'de groupe', 'par aménagement du milieu', 'éducation nouvelle', etc. Ces approches soulignent que l'enfance gagne la confiance du monde adulte. On lui donne son autonomie et on lui permet de prendre des initiatives. La soumission et la passivité de l'élève font place à l'autonomie et à l'activité.

Le travail en groupe prend une signification de premier ordre. La pédagogie est dite 'active'. «Il s'agit donc de susciter des taches ou l'élève ne sera pas un simple exécutant. Il va entreprendre des démarches, des recherches personnelles; le travaille ne lui sera pas imposé, les étapes ne sont pas prescrites »selon (Snyders, 1975, p.59).

\section{- La globalisation}

La Gestal théorie confirme les travaux de Olivier Decroly en 1929 portant sur la globalisation de l'enseignement. Le 'tout' est préféré aux 'parties'. C'est la méthode globale (opposée à la méthode 
analytique) qui amène l'élève progressivement de la phrase vers le mot et, enfin, à la lettre.

Snyders (1975), était l'un des pédagogues à avoir refusé de donner le primat aux théories nouvelles sur l'éducation traditionnelle, tente de jeter un pont entre les deux courants. «A partir des définitions, éclaircissements et confrontations des termes en présence, notre seconde prétention va jusqu'à penser qu'une voie peut s'ouvrir : une pédagogie moderne qui ferait la synthèse du traditionnel et du nouveau, synthèse et pas confusion » (p. 9).

Dans la même perspective, Debesse (1955) reconnaît des avantages aussi bien à l'enseignement traditionnel qu'aux méthodes nouvelles : «L'enseignement traditionnel accorde une grande place à la transmission du savoir par le maître et par le livre, mais il ne réduit pas pour autant l'élève à une fonction passive d'enregistrement. De leur coté, si les méthodes nouvelles font plus largement appel aux activités de recherche et de création personnelle et s'écartent le plus possible des procédures de pure mémorisation, elles ne peuvent rejeter tout enseignement proprement dit »(p.817). 
Revue des sciences de l'homme et de la société.. Dr. Tarek Saker, Dr : Said Mezroua

\section{Conclusion:}

Le foisonnement de recherches, d'écrits, d'analyses sur la pédagogie témoigne de la préoccupation de l'homme universel sur le sujet. Qu'il en soit ainsi n'a rien d'extraordinaire si l'on considère que l'éducation est le processus fondamental par lequel l'être humain crée, développe, maintient et pérennises ses valeurs. 


\section{Références}

1 - Château, J. (1968). Autour de l'élève. Paris : Ed. Vrin.

2- Claparade, E. (1973). L'éducation fonctionnelle. Neuchatel : Ed. Delachaux et Niestle.

3- Decroly, O. (1929). La fonction de globalisation et l'enseignement. Bruxelles : Ed. Lamertin

4- Debesse, M. (1955). La formation pédagogique-traité de psychologie appliquée. Paris : Ed. P.U.F.

5- Dewey, J. (1967). L'école et l'enfant. Neuchatel : Ed. Delachaux et Niestle.

6- Durkheim, E. Penseurs de l'éducation perspective : revue trimestrielle de l’Education. Vol. XXIII, Nº 1-2;1993, p. 313.

7- Equipe pédagogique. (1981). Démarche d'éveil. Paris : Revue de l'E.P.S., 172, pp. 57-79.

8- Gabaude, J.M. (1972). La pédagogie contemporaire. Paris: Ed. Privat.

9- Gilbert, R. (1980). Bon pour enseigner. Liège : Ed. Mardaga.

10- Hebrard, A. (nd). Recherche et pédagogie du sport et de l'éducation physique en France. Edition revue EPS. pp.25-36.

11- Louanchi, D. (1987). Eléments de pédagogie. Alger : Ed. OPU.

12- Montessouri, M. (1952). Pédagogie scientifique. Paris : Ed. Desclée de Brauwers.

13- Paquette, C. (1979). Vers une pratique de la pédagogie ouverte. Paris : Ed. Vuibert.

- Rogers, C. R. (1984). Liberté pour apprendre. Paris : Ed. Dunod. 
Revue des sciences de l'homme et de la société.. Dr. Tarek Saker, Dr : Said Mezroua

14- Snyders, G. (1975). Pédagogie progressiste-éducation traditionnelle ou nouvelle. Paris : Ed. P.U.F.

15- Thoraille, R. (1968). Psycho-pédagogie. Strasbourg: Ed. ISTRA. 